Notes

\title{
Separation of Atomic Vapor of Lead in Direct Sampling of Urine with a Separative Column Atomizer
}

\author{
Masaaki Yanagisawa ${ }^{\dagger}$ Kazuo KatoH and Kuniyuki Kitagawa \\ Department of Synthetic Chemistry, Faculty of Engineering, Nagoya University, Nagoya 464-01, Japan
}

\begin{abstract}
Keywords Atomic lead vapor, atomic absorption spectrometry, urine, separative column atomizer, direct urine analysis, background absorption, direct atomization
\end{abstract}

In a atomic absorption spectrometry with electrothermal atomization (ETA-AAS), biological materials such as urine ${ }^{1}$, blood and tissues are usually analyzed after ashing in order to remove matrices at a low temperature. In the ashing process, however, loss of lead has been often encountered. This loss is attributed to relatively high vapor pressures of lead species: metal, oxides, chlorides and sulfides. Accordingly, direct atomization is preferable for the determination without the analyte loss. In the direct determination of lead in urine without any pretreatments, large amounts of inorganic matrix constituents such as alkali halides are vaporized simultaneously at the appearance temperature of atomic lead vapor. This causes interferences with atomic absorption measurements; background absorption and chemical interference are encountered. ${ }^{2}$ Background absorption is also caused by pyrolytically evolved species such as smoke and diatomic molecules (NO, CO, SO etc.). Problems associated, with both background absorption and chemical interference can be overcome through the separation between atomic and molecular vapors.

In previous studies ${ }^{3-6}$, the effectiveness of separative column atomizers (SCA's) has been proved for the separation between atomic vapor of mercury or cadmium; in these procedures, diatomic molecular species such as $\mathrm{CO}$ and $\mathrm{NO}$ evolved on direct atomization of biological samples. In this study, an improved separative column atomizer was successfully applied to cause the separation between atomic vapor of lead and vapor due to molecular species on the direct atomization of urine.

\section{Experimental}

The atomic absorption system used was a Nippon Jarrell Ash JE-25 (Czerny-Turner mount) equipped with an SCA. The SCA was basically the same as those constructed for the previous study. ${ }^{4}$ An improvement was made in order to attain higher column temperatures;

$\dagger$ Present address; Research and Development Laboratory, Japan Fine Ceramics Center, Mutsuno, Nagoya 456, Japan $1530^{\circ} \mathrm{C}$ in the middle of the column, $1420^{\circ} \mathrm{C}$ at the atomization site and $1450^{\circ} \mathrm{C}$ at the column outlet end where AA measurements were made. The column was a recrystallized alumina tube (Nippon Kagaku Togyo Co. SSA-S) of $10 \mathrm{~mm}$ i.d., $15 \mathrm{~mm} \mathrm{o.d.} \mathrm{and} 200 \mathrm{~mm}$ in length, packed with granules $(0.25-0.5 \mathrm{~mm}$ in diameter) of graphite, glassy carbon (Tokai carbon Co.), activated charcoal or alumina. The packing length was $60 \mathrm{~mm}$. The column was heated in a tubular $\mathrm{SiC}$ heating element 4,5 in order to attain as high a temperature as $1500^{\circ} \mathrm{C}$. The column temperatures were measured with 3 thermocouples (13PR). An elliptical loop of a tungsten wire of $0.8 \mathrm{~mm}$ in diameter and $20 \mathrm{~mm}$ in length, as a sample holder, was attached to a molybdenum rod of $2.5 \mathrm{~mm}$ in diameter, which was manipulated for the sample introduction. For atomic and background absorption measurements, the light beam from a hollow cathode or a $\mathrm{D}_{2}$ lamp was passed $3 \mathrm{~mm}$ below the column outlet end.

Urine samples were prepared by adding thiourea or EDTA for matrix modification ${ }^{7}$ and improvement of sensitivity. An aliquot $(5 \mu \mathrm{l})$ of a urine sample was taken onto the tungsten(W)-loop by means of a micropipet (Gilson Co.), dried sufficiently with an electric fan heater. The loop was set into the sample introduction head which was connected to the column top. After a steady carrier gas flow rate (180$300 \mathrm{ml} / \mathrm{min}$ ) was established for one minute, the $\mathrm{W}$ loop was manually inserted into the atomization site $20 \mathrm{~mm}$ above the packing layer. While passing through the packing layer toward the column outlet end, the pyrolytically resulting species underwent reactions to attain further decomposition and gas chromatographic separation. A successive run was made after cooling the W-loop sufficiently. Cycle time required for one run was ca. $10 \mathrm{~min}$.

\section{Results and Discussion}

Figure 1(a) shows typical AA traces obtained with SCA's packed with different materials. The highest sensitivity is obtained with the glassy carbon-packed 
SCA. The sharpest AA peak without tailing is yielded with the graphite-packed one. Notable peak tailing is observed with the activated charcoal-packed SCA. Complex peaks are caused with alumina-packed SCA. Accordingly, graphite was selected as the packing material; with it, the best separation was attained at the sacrifice of some sensitivity. The reproducibility in repeated measurements was dependent on the packing length. Four different packing lengths; 40, 60, 150 and $200 \mathrm{~mm}$, were tested. A good reproducibility $(<5 \%$ in relative standard deviation) was obtained for the shorter lengths, 40 and $60 \mathrm{~mm}$. On the other hand, the peak height decreased gradually during 24 repeated runs with the longer packings of 150 and $200 \mathrm{~mm}$. The separation between atomic and molecular species was inadequately obtained with the shortest packing of $40 \mathrm{~mm}$. The packing length of $60 \mathrm{~mm}$, therefore, was used as the optimum. The poor reproducibility found for the longer packings might be associated with a large temperature distribution along the column.

Figure 1(b) shows atomic and background absorption traces obtained with the graphite-packed SCA. Trace A was obtained for a lead standard solution with a hollow cathode lamp (HCL) as the light source, and trace $\mathrm{C}$ was observed on the direct atomization of a urine sample with a $\mathrm{D}_{2}$-lamp. The former indicates a single AA peak caused by lead atoms and the latter two background peaks. Trace $B$ was obtained for a urine solution spiked with an aliquot $(2.5 \mathrm{ng})$ of a standard

(a)

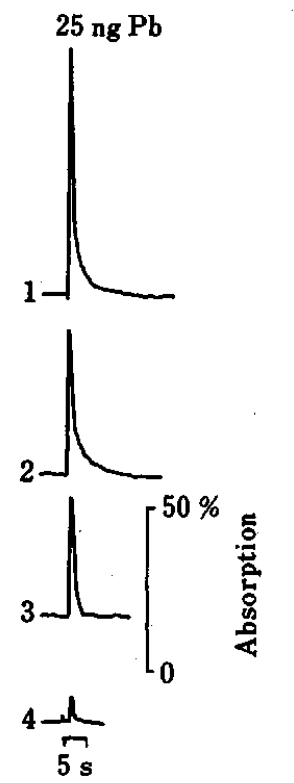

(b)

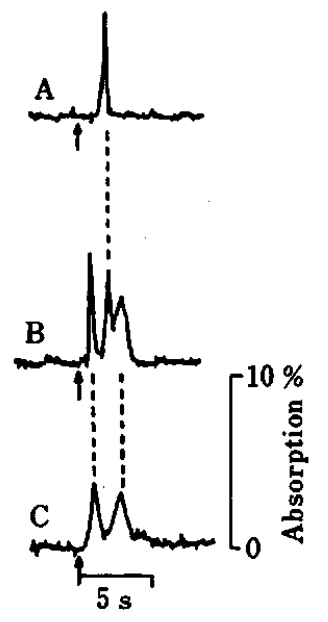

Fig. 1(a) Typical absorption peak traces observed through an SCA. 1, absorption peak with glassy carbon-packed SCA and a Pb-hollow cathode lamp (HCL); 2, activated charcoal; 3, graphite; 4 , alumina.

Fig. 1(b) A, standard solution (10 $\mathrm{ng} \mathrm{Pb}$ ) of lead containing EDTA/Pb-HCL; B, a urine sample spiked with a lead standard solution (2.5 $\mathrm{ng} \mathrm{Pb}$ )/Pb-HCL; C, the urine sample (same as that used for the middle trace)/ $D_{2}$ lamp. lead solution. Three absorption peaks appear almost separated. The appearance time of the second peak in trace B coincides with that in trace A. On the other hand, the appearance times of the first and third peaks are almost the same for the $B$ and the $C$ traces. Consequently, the second peak for the urine sample is due to atomic absorption by lead atoms, whereas the first and third peaks are caused by background absorptions. The species which are most likely responsible for the background absorptions are alkali haildes such as $\mathrm{NaCl}$ and $\mathrm{KCl}$ and diatomic molecules such as $\mathrm{NO}$ and CO.8,9 Alkali halides, which are major matrix components in urine, have broad absorption spectra in UV and visible regions.,9 Alkali halides may be responsible for the third background absorption peak, since they have a lower vapor pressure than that of $\mathrm{Pb}$. In the previous studies ${ }^{3,4,6}$, such diatomic species as NO and $\mathrm{CO}$, possessing strong bands in UV region, were found to cause background absorption in the direct atomization of organic matrices containing $\mathrm{N}$ and $\mathrm{C}$. Thus, the first background peak may be caused by NO and/or CO.

In this study, an improved SCA capable of attaining as high a temperature as $1530^{\circ} \mathrm{C}$ was successfully applied to the gas chromatographic separation of atomic vapor of lead from molecular species such as alkali halides, $\mathrm{CO}$ and NO, which caused background absorptions on the direct atomization of a urine sample. Further improvements, to elevate the column temperature and to lessen its distribution, are necessary for more complete separation and higher sensitivity.

The authors wish to thank Prof. S. Tsuge for helpful advice, and Mr. S. Takahashi, Mr. T. Imura and Mr. T. Watanabe for elaborately manufacturing the SCA.

\section{References}

1. K. S. Subramanian, Prog. Anal. At. Spectrosc., 11, 511 (1988).

2. B. Welz, S. Akman and G. Schlemmer, J. Anal. At. Spectrom., 2, 793 (1987) (Anal. Ab., 50, 3B137, 1988).

3. M. Yanagisawa, H. Suzuki, K. Kitagawa and S. Tsuge, Spectrochim. Acta, 37B, 493 (1983).

4. M. Yanagisawa, A. Itoh and K. Kitagawa, J. Spectrosc. Soc. Jpn., 37, 446 (1988).

5. K. Kitagawa, T. Takeuchi and M. Yanagisawa, Anal. Sci., 5, 445 (1989).

6. M. Yanagisawa, K. Ida and K. Kitagawa, Anal. Sci., 5, 765 (1989).

7. J. Ebert and H. Jungmann, Fresenius Z. Anal. Chem., 272, 287 (1974).

8. H. Massmann, Talanta, 29, 1051 (1982).

9. H. Massmann, Z. EL. Gohary and S. Gucer, Spectrochim. Acta, 31B, 399 (1976).

(Received December 1, 1989)

(Accepted February 24, 1990) 